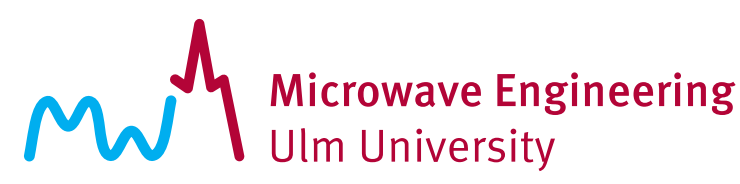

\section{Clustering of Closely Adjacent Extended Objects in Radar Images using Velocity Profile Analysis}

Johannes Schlichenmaier, Fabian Roos, Philipp Hügler, and Christian Waldschmidt

Institute of Microwave Engineering, Ulm University, 89081 Ulm, Germany Email: schlichenmaier@ieee.org (c) 2019 IEEE. Personal use of this material is permitted. Permission from IEEE must be obtained for all other uses, in any current or future media, including reprinting/republishing this material for advertising or promotional purposes, creating new collective works, for resale or redistribution to servers or lists, or reuse of any copyrighted component of this work in other works. 


\title{
Clustering of Closely Adjacent Extended Objects in Radar Images using Velocity Profile Analysis
}

\author{
Johannes Schlichenmaier, Fabian Roos, Philipp Hügler, and Christian Waldschmidt \\ Institute of Microwave Engineering, Ulm University, 89081 Ulm, Germany \\ Email: schlichenmaier@ieee.org
}

\begin{abstract}
As high resolution automotive radars become more common, so does their usage for next-generation functionalities like advanced driver assistant systems and autonomous driving. This creates the need for robust clustering techniques to distinguish among multiple extended objects like vehicles in the same scenario. One especially challenging scenario is that of separating two extended targets close to each other, each following its own trajectory. This paper proposes a clustering algorithm based on the analysis of the velocity profile to divide target points of multiple vehicles into sub-clusters. The theoretical background is explained and shown on simulation data. The algorithm is verified using radar measurements of two extended vehicular targets.
\end{abstract}

\section{INTRODUCTION}

In recent years, capabilities of automotive radars have increased substantially. In [1], a high-resolution radar is used to classify road users. In [2] an experimental radar system is shown, that is able to detect dozens of target points per road user in an automotive setting. These high performance radars pave the way for new radar applications in the field of advanced driver assistance systems and autonomous driving, by allowing for estimating the dimensions and orientation of road users in a scenario [3]. The estimation of motion parameters like velocity and yaw rate using one or more radar sensors was shown as well [4], [5], [6].

An integral part of each of these signal processing algorithms for extracting properties of radar objects is the reliable assignation of the measured target points to one or more objects. Usually, due to its excellent performance and the minimal a priori knowledge being necessary, Density-Based Spatial Clustering of Applications with Noise (DBSCAN) [7] or one of its derivates is used for this task.

The clustering of radar data is usually done either in the Cartesian domain, e.g., with a dynamically self-configuring approach based on the density of the cluster [8] or in the range-DoA domain to accommodate for the operating principle of radar data [9]. This modification is extended in [10] to include the dimension of velocity and [11] proposes a direct application on the measured data in range, velocity, and direction of arrival (DoA), with different radii for each domain dependent on the radar system parameters.

The inclusion of the velocity domain in the clustering process allows for separating spatially close objects, that are moving in a way so that the resulting target points exhibit a noticeably different radial velocity. In certain cases this makes it possible to only rely on difference in radial velocity to distinguish multiple objects. But in many cases, the velocity of vehicles as measured by the radar sensor is very similar, e.g., if the vehicles are driving in a convoy, rendering this approach impossible. In [12] a technique is shown to individually track multiple extended radar objects. This approach relies on setting up a track of the object as well as the vehicles moving with distinctively different velocities.

In this paper, an approach is shown to distinguish spatially close objects with similar but different motion, each following its own trajectory. This approach is based on the analysis of the velocity profile of a misclustered target list set, which due to the spatial adjacency of the objects - includes target points belonging to both objects. This makes it possible to repair the original cluster by dividing it up into sub-clusters which contain only target points most likely to belonging to one object. The necessary theoretical background is given and illustrated by a simulation. The proposed algorithm chain to solve the problem is presented and explained. Finally the algorithm chain is validated using real radar measurements.

\section{Velocity Profile Analysis}

To better understand the underlying idea of the algorithm, it is helpful to recall the way, the measured radial velocity $v_{\mathrm{r}, i}$ of a single radar target point $i$ originating at the surface of a moving, extended target, i.e., a vehicle, depends on the DoA of the target point $\theta_{i}$, as well as the motion of the vehicle [5]. For a linear motion of the vehicle, described by its velocity vector $\left(v_{\mathrm{x}}, v_{\mathrm{y}}\right)$, this dependency is shown in Eq. (1), if a single sensor is used. Similarly, if the vehicle is moving in a nonlinear fashion, i.e., a turn, $v_{\mathrm{r}, i}$ depends not on the velocity vector, but instead on the yaw rate of the vehicle $\omega$ and the turning center $\left(x_{\mathrm{ICR}}, y_{\mathrm{ICR}}\right)$, as shown in Eq. (2).

$$
\begin{array}{lll}
v_{\mathrm{r}, i}=v_{\mathrm{x}} & \cos \left(\theta_{\mathrm{i}}\right)+v_{\mathrm{y}} & \sin \left(\theta_{i}\right) \\
v_{\mathrm{r}, i}=\underbrace{\omega y_{\mathrm{ICR}}}_{C} & \cos \left(\theta_{i}\right)+\underbrace{\left(-\omega x_{\mathrm{ICR}}\right)}_{S} & \sin \left(\theta_{i}\right)
\end{array}
$$

In both equations, the prefactors of the trigonometric functions can be substituted by two generic motion parameters $C$ and $S$. If the radial velocities $v_{i}$ of multiple target points belonging to a single vehicle are plotted versus their respective DoA as $\sin \left(\theta_{i}\right)$ in a so called velocity profile analysis (VPA) diagram, the points mostly align in a linear fashion, which can be described by the generic motion parameter set $(C, S)$. 


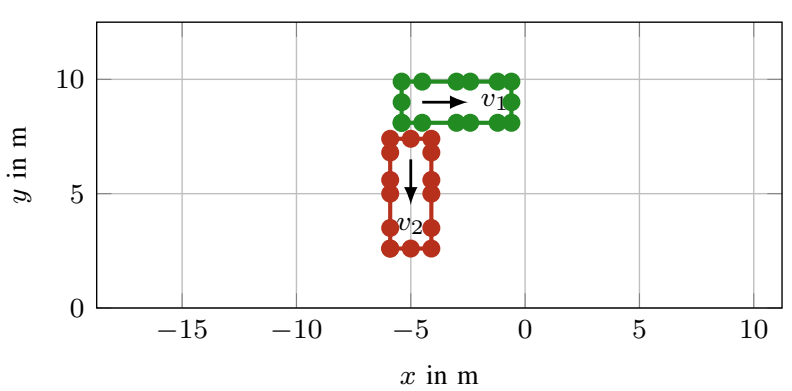

(a) Cartesian plot.

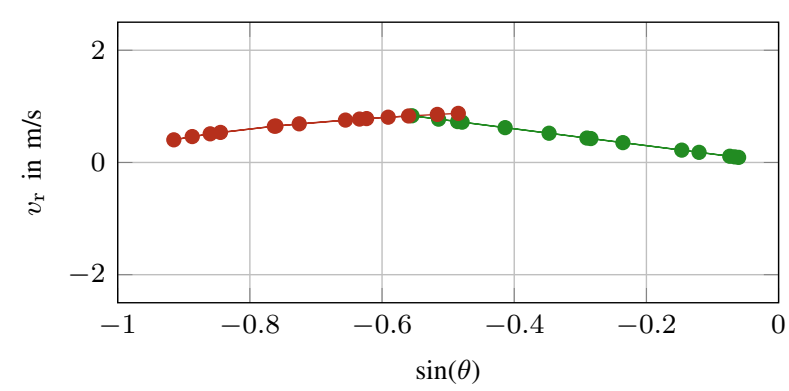

(b) velocity profile analysis plot.

Fig. 1. Radar simulation of two vehicles close together with similar velocities but different trajectories. The radar sensor is placed at $\left(x^{\mathrm{S}}, y^{\mathrm{P}}\right)=(0,0)$.

This is shown in Fig. 1, where two extended radar objects with similar velocities of $v_{1}=1.5 \mathrm{~m} / \mathrm{s}$ and $v_{2}=1 \mathrm{~m} / \mathrm{s}$, but different trajectories, are simulated with 14 target points each placed on their surfaces.

The resulting VPA plot can be seen in Fig. 1(b). While a clustering algorithm considering range, DoA, and even velocity will most likely group all those target points into a single cluster, it can be clearly seen that each point set is aligned on its own straight line described by their respective $(C, S)$ sets. This makes it possible to separate them, at least partially, if the two $(C, S)$ sets can be determined from the target points, which forms the basis for the proposed algorithm.

\section{Sub-Clustering USing Velocity Profiles}

To use the motion information to sub-cluster a mis-clustered set containing target points of multiple moving objects, the $(C, S)$ sets have to be extracted first from the VPA. This is done with a series of steps outlined in [4].

To accomplish this, first, a RANdom SAmple Consensus (RANSAC) algorithm is used to find the first main direction model of the targets. RANSAC will try to find the most likely linear model of a set of points by randomly choosing two sample points and counting the number of inlier points which are located within a certain corridor around the direct connection between those two points. This is repeated a set number of times after which the model with the highest number of inliers is chosen. As the algorithm has to prevent detecting too many target points of both vehicles as inliers at once, the corridor has to be chosen very narrow. After extracting this first assumption, the model can be refined by linearly optimizing the distance of the inliers to the model.

With the extracted model, the target points belonging to the vehicle have to be selected for a new sub-cluster. Besides the inlier points, this concerns the target points created by the wheels of the vehicle. These present themselves in the VPA as a chain of target points with very similar DoA but greatly differing velocity due to the different wheel parts moving within a velocity range of $0 \leq v_{\text {wheel }} \leq 2 v_{\text {car }}$.

These wheel target points can be selected for the new subcluster for the vehicle by a proposed solution called wheel enveloping. Because the wheel target points are only visible, if the main motion model has a noticeable extend in the DoA domain, this method consists of dividing the angular range,

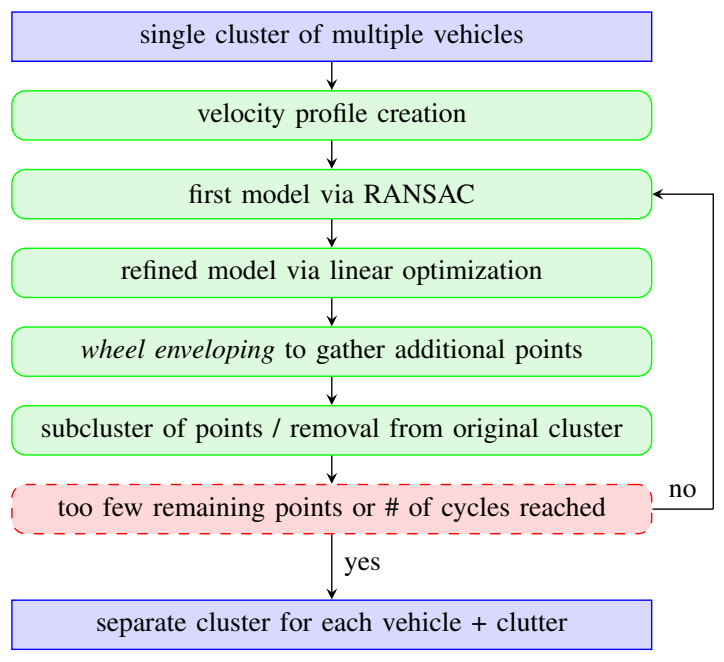

Fig. 2. Algorithm chain for the velocity profile based sub-clustering.

which is occupied by the inliers of the model into sub-ranges. For every sub-range, a search for a chain of target points in velocity-direction is performed, which is originating from the model-based velocity value of this sub-range in both directions and whose links are not more than a specified velocity value $\Delta v_{\max }$ away from each other. At the first occurrence of a velocity difference greater than $\Delta v_{\max }$ the chain is interrupted and the remaining target points are not further taken into account.

After the wheel enveloping, the points in the new subcluster are removed from the original cluster. As shown in the overview chart in Fig. 2, the process repeats - starting again with the RANSAC step - until either the desired number of iterations is reached or too few points are left to successfully perform a motion model extraction. The remaining points form their own cluster of points classified as clutter.

\section{Measurement Data Characteristics}

To verify the proposed sub-clustering algorithm, measurements were performed with a $77 \mathrm{GHz}$ experimental radar sensor [13] operating in time-division multiplexing (TDM) multiple-input multiple-output mode. Its three transmitters and eight receivers form a uniform linear array with $\frac{\lambda}{2}$ spacing without overlapping virtual elements. The motion-induced 


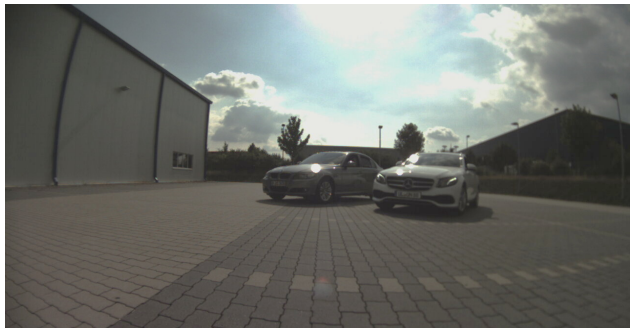

(a) Picture of the scenario.

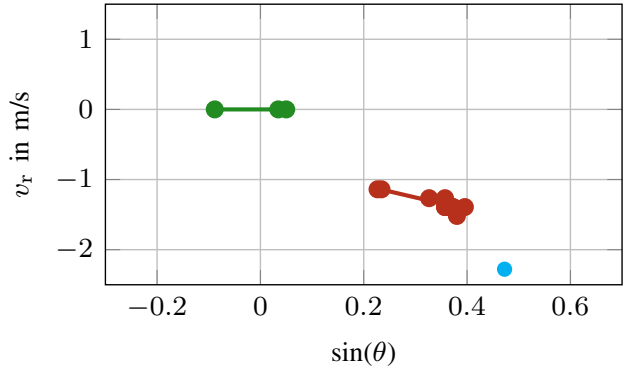

(b) Velocity profile analysis plot.

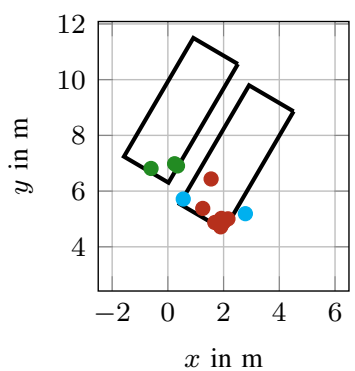

(c) Sub-clustered target points.

Fig. 3. Scenario A: Both cars (-) are oriented diagonally. The wheels are not visible in the radar in. The algorithm assigns the target points to the stationary (๑), the moving car $(\bullet)$, or neither $(\bullet)$.

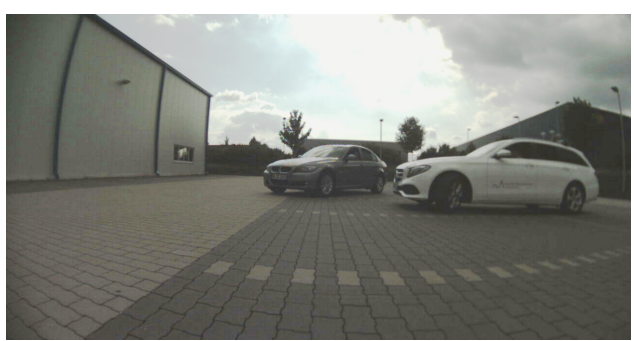

(a) Picture of the scenario.

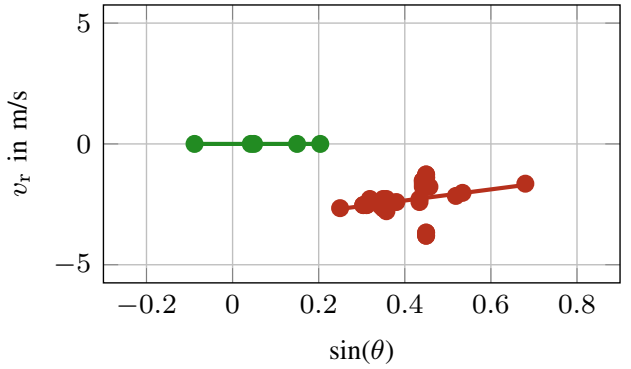

(b) Velocity profile analysis plot.

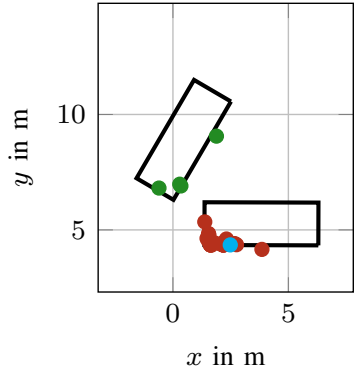

(c) Sub-clustered target points.

Fig. 4. Scenario B: The moving car is laterally oriented with respect to the radar. The front wheel is clearly visible. The algorithm assigns the target points to the stationary $(\bullet)$, the moving car $(\bullet)$, or neither $(\bullet)$.

phase error which leads to errors in the DoA estimation is compensated as described in [14].

The TDM usually reduces the maximum unambiguous velocity, which is restored by evaluating the phase trend along the virtual aperture as described in [15]. The used bandwidth of $2 \mathrm{GHz}$ leads to a range resolution of $7.5 \mathrm{~cm}$. In total 258 frequency chirps are transmitted and with a chirp duration of $50 \mu$ s and a chirp repetition time of $60 \mu$ s this results in a velocity resolution of $0.13 \mathrm{~m} / \mathrm{s}$.

For the scenario, two cars were measured in front of the sensor. One of them was oriented at an angle toward the sensor and remained stationary throughout the measurement. The other car was equipped with a global navigation satellite system to obtain a ground truth information. It was driven around and close to the stationary car.

\section{Measurement Evaluation}

From the available measurement data, several snapshots were selected in which both cars were close spatially as well as in velocity. The target lists of these snapshots were first subjected to a very rough clustering with a simple DBSCAN using a high search radius of $\varepsilon=3 \mathrm{~m}$, to accommodate for the fact that stationary objects do not yield too many target points as moving ones. The resulting cluster was subjected to the algorithm chain with maximum loop iterations of $N=2$.

Scenario A (Fig. 3) was selected because both the moving and stationary car are oriented in a similar fashion. The moving car is currently in the process of accelerating toward the radar sensor. The front of the car is visible on the radar image.

Fig. 3 (b) shows the VPA diagram with colors being assigned after the sub-clustering chain. The target points of the original cluster are colored to show them being detected as part of the stationary car $(\bullet)$, the moving car $(\bullet)$, or neither of them (possible clutter, $\bullet$ ). The straight lines of the same colors as the target points represent the model extracted from them.

Fig. 3 (c) shows the target points of the VPA plot in Cartesian coordinates, coded in their corresponding colors, together with the ground truth position of both cars (-).

The algorithm is successfully able to separate both cars. Two target points belonging to the moving car do not support the main motion model and are assigned to be clutter.

Fig. 4 shows scenario $\mathrm{B}$, where the moving car is oriented laterally to the radar sensor, making a turn towards the radar. Mostly the front and left side of the car is visible in the radar image. The targets of the front wheel are clearly visible.

Besides the target points of the main motions of both cars, Fig. 4(b) shows the distinctive chain of targets originating from the wheel. As can be seen, the wheel enveloping algorithm is working correctly to assign the wheel target points to the moving car while still being able to correctly extract the main motion model. This can also be seen in the Cartesian plot $n$ Fig. 4(c). A single target point was classified as not belonging to either car and as such as clutter, which is not displayed in the range of the VPA diagram. 


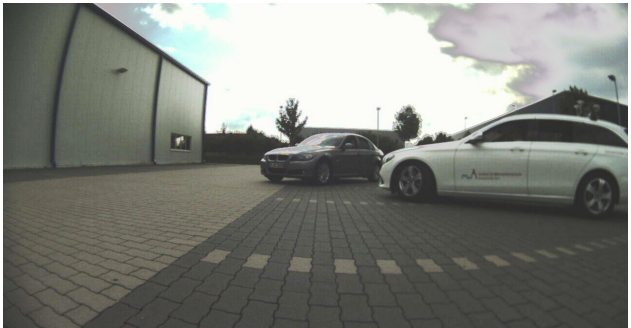

(a) Picture of the scenario.

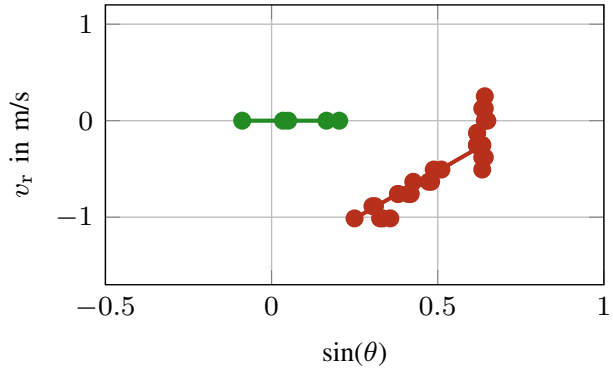

(b) Velocity profile analysis plot.

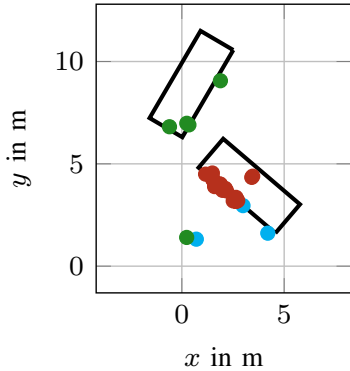

(c) Sub-clustered target points.

Fig. 5. Scenario C: The moving car is orthogonally aligned with respect to the stationary one. The front wheel is clearly visible. The algorithm assigns the target points to the stationary $(\bullet)$, the moving car $(\bullet)$, or neither $(\bullet)$.

In the third selected scenario C (Fig. 5) the moving car is orthogonally aligned to the stationary car. It just starts to drive backwards, away from the stationary car. The front wheel and the left side of the moving car is visible in the radar image.

The associated VPA diagram (Fig. 5 (b)) shows again the clear assignation of the target points to their respective cars. Again, the wheel enveloping algorithm successfully assigns the wheel target points to the moving car.

This scenario is particularly challenging because velocities of target points from both vehicles overlap. Nevertheless, the algorithm is mostly able to correctly assign the target points to their respective objects, as can be seen in Fig. 5 (c). Two target points from the moving car are classified as not belonging to either, but are also extreme outliers in the VPA diagram outside of the plotted range. The target point falsely assigned to the stationary car $(\bullet)$ can easily be removed from the other target points due to its euclidean distance to the other points of the sub-cluster.

\section{CONCLUSION}

The proposed algorithm on the basis of extracting multiple velocity profile models is able to divide a mis-clustered target point set containing points belonging to different vehicles into smaller sub-clusters containing only points of a single vehicle. It is able to correctly perform a secondary sub-clustering for closely adjacent extended radar objects, like cars, with similar velocities but different trajectories, without the need for too cautious clustering parameters. The wheel enveloping sub-algorithm is correctly detecting target points belonging to the wheels of the car and groups them together with their respective main motion components. Some target points get mis-assigned to either a stationary vehicle, or dismissed as clutter, the former of which can usually be easily detected because of their large distance the rest of the points. As such the algorithm proves to be an effective secondary step after a first rough clustering to repair mis-shaped clusters for the subsequent mid-level signal processing steps.

\section{ACKNOWLEDGMENT}

This work was supported by the German Federal Ministry of Education and Research (BMBF) within the framework of the KoRRund project (Grant 16ES0580).

\section{REFERENCES}

[1] E. Schubert, F. Meinl, M. Kunert, and W. Menzel, "High Resolution Automotive Radar Measurements of Vulnerable Road Users - Pedestrians \& Cyclists," in International Conference on Microwaves for Intelligent Mobility, 2015.

[2] F. Meinl, M. Stolz, M. Kunert, and H. Blume, "An experimental high performance radar system for highly automated driving," in International Conference on Microwaves for Intelligent Mobility, 2017.

[3] F. Roos, D. Kellner, J. Klappstein, J. Dickmann et al., "Estimation of the Orientation of Vehicles in High-Resolution Radar Images," in International Conference on Microwaves for Intelligent Mobility, 2015.

[4] D. Kellner, M. Barjenbruch, K. Dietmayer, J. Klappstein, and J. Dickmann, "Instantaneous lateral velocity estimation of a vehicle using Doppler radar," in Proceedings of the 16th International Conference on Information Fusion, 2013.

[5] D. Kellner, M. Barjenbruch, J. Klappstein, J. Dickmann, and K. Dietmayer, "Instantaneous full-motion estimation of arbitrary objects using dual Doppler radar," in Intelligent Vehicles Symposium Proceedings, 2014.

[6] J. Schlichenmaier, L. Yan, M. Stolz, and C. Waldschmidt, "Instantaneous Actual Motion Estimation with a Single High-Resolution Radar Sensor," in International Conference on Microwaves for Intelligent Mobility, 2018.

[7] M. Ester, H.-P. Kriegel, J. Sander, and X. Xu, "A Density-Based Algorithm for Discovering Clusters in Large Spatial Databases with Noise." in Proceedings of the Second International Conference on Knowledge Discovery and Data Mining, vol. 96, 1996.

[8] J. Schlichenmaier, F. Roos, M. Kunert, and C. Waldschmidt, "Adaptive Clustering for Contour Estimation of Vehicles for High-Resolution Radar," in International Conference on Microwaves for Intelligent Mobility, 2016.

[9] D. Kellner, J. Klappstein, and K. Dietmayer, "Grid-based DBSCAN for clustering extended objects in radar data," in Intelligent Vehicles Symposium), 2012.

[10] T. Wagner, R. Feger, and A. Stelzer, "Modification of DBSCAN and application to range/Doppler/DoA measurements for pedestrian recognition with an automotive radar system," in European Radar Conference, 2015.

[11] M. Li, M. Stolz, Z. Feng, M. Kunert et al., "An Adaptive 3D Grid-Based Clustering Algorithm for Automotive High Resolution Radar Sensor," in International Conference on Vehicular Electronics and Safety, 2018.

[12] A. Scheel and K. Dietmayer, "Tracking Multiple Vehicles Using a Variational Radar Model," Transactions on Intelligent Transportation Systems, 2018.

[13] P. Hügler, F. Roos, M. Schartel, M. Geiger, and C. Waldschmidt, "Radar Taking Off: New Capabilities for UAVs," IEEE Microwave Magazine, vol. 19 , no. 7, pp. 43-53, Nov. 2018.

[14] J. Bechter, F. Roos, and C. Waldschmidt, "Compensation of MotionInduced Phase Errors in TDM MIMO Radars," Microwave and Wireless Components Letters, 2017.

[15] F. Roos, J. Bechter, N. Appenrodt, J. Dickmann, and C. Waldschmidt, "Enhancement of Doppler Unambiguity for Chirp-Sequence Modulated TDM-MIMO Radars," in International Conference on Microwaves for Intelligent Mobility, 2018. 